\title{
Gravitational Instabilities in Circumstellar Disks and the Formation of Binary Companions
}

\author{
FRED C. ADAMS \\ Physics Department, University of Michigan, Ann Arbor, MI 48109, USA \\ WILLY BENZ \\ Steward Observatory, University of Arizona, Tucson, AZ 85721, USA
}

\begin{abstract}
We review recent work on gravitational instabilities in circumstellar disks and discuss implications for the possibility of forming binary companions within these disks. Linear stability analysis indicates that self-gravitating modes with asimuthal wavenumber $m=1$ will grow strongly in star/disk systems with the physical properties observed in astrophysical systems. Smooth particle hydrodynamic simulations indicate that these self-gravitating instabilities (with $m=1$ and higher) will grow into the nonlinear regime. In many cases, a spiral arm will collapse into a gravitationally bound "knot" of gas; these knots typically contain $1 \%$ of the mass of the disk and travel on elliptical orbits about the central star.
\end{abstract}

\section{INTRODUCTION}

Over the course of the last decade, a new and reasonably successful paradigm of the star formation process has emerged (see, e.g., Shu et al. 1987; Lada \& Shu 1990). The main shortcoming of the current theory is its failure to properly account for the formation of binaries: most stars are observed to live in binary systems (e.g., Abt 1983), but the current theory deals directly with only the formation of single stars. However, both theory and observations now indicate strongly the presence of disks associated with young stellar objects (cf. the reviews of Appenzeller \& Mundt 1989; Bertout 1989; Shu et al. 1987), although the exact properties of such disks remain controversial. The available evidence (see, e.g., Adams et al. 1989a; Adams et al. 1988) indicates that these disks may produce significant luminosity $\left(L_{D} \sim L_{*} \sim 1 \mathrm{~L}_{\odot}\right)$ and have moderate masses $\left(M_{D} \sim M_{*} \sim 1 \mathcal{M}_{\odot}\right)$. For systems with $M_{D} \sim M_{*}$, the idea of forming a binary companion within the disk provides an intriguing possibility. In this study, we consider the growth of global gravitational instabilities in star/disk systems; the hope is that these instabilities can grow into the nonlinear regime and form stellar objects within the disk. We have studied the behavior of $m=1$ modes both numerically (Adams et al. 1989b; hereafter ARS) and analytically (Shu et al. 1990; hereafter STAR). We find good agreement between the two approaches. We have also studied the nonlinear growth of these instabilities through numerical simulations using smooth particle hydrodynamics (Benz \& Adams 1992). In this present discussion, we summarize our current understanding of these instabilities. 


\section{LINEAR STABILITY ANALYSIS OF $M=1$ MODES}

\subsection{The Initial (Unperturbed) State}

Since the overall goal of the linear study is to determine the growing normal modes of a star/disk system, we begin by specifying the basic unperturbed state. The physical system consists of a star and an accompanying gaseous disk. The growth of sprial modes is mainly determined by three elements: self-gravity, pressure, and differential rotation. The gravitational forces are determined by the potential of the star and by the disk's surface density distribution $\sigma_{0}(r)$, which we take to be a simple power-law in radius $r$ from the central star (the disk is also assumed to be infinitesimally thin and in centrifugal equilibrium). The pressure is determined by the temperature distribution, or equivalently, the distribution of sound speed in the disk; we take the sound speed $a(r)$ to be a power-law in radius. The rotation curve $\Omega(r)$ in the disk is then determined self-consistently from the potential of the star, the potential of the disk, and the pressure gradients. Since the potential well of the star dominates that of the disk everywhere except near the disk's outer edge, the rotation curve is nearly Keplerian throughout most of the disk's radial extent. (For thin gaseous disks, the pressure gradients are small compared to the gravitational forces and do not significantly affect the rotation curve). Finally, we must specify the radial extent of the disk; since observations indicate that 'typical' disk sizes are approximately $100 \mathrm{AU}$ (e.g., Edwards et al. 1987), we consider disk sizes $R_{D}$ up to $10^{4}$ times the radius $R_{*}$ of the star (only the ratio $R_{D} / R_{*}$ enters into the calculations).

\subsection{Modes with Azimuthal Wavenumber $m=1$}

Our study concentrates on modes with azimuthal wave number $m=1$, since these modes can be global in extent and may also be the most difficult modes to suppress in unstable gaseous disks. Modes with $m=1$ correspond to elliptic streamlines (i.e. eccentric particle orbits), a special characteristic of Keplerian potentials. Thus, for a disk with an exact Keplerian rotation curve and no interactions between particles, $m=1$ disturbances correspond to purely kinematic modes of the system; for realistic disks (with pressure), a relatively 'small' amount of self-gravity is required to 'hold the mode together' and sustain its growth.

One unique and important aspect of $m=1$ modes is that the center of mass of the perturbation in the disk does not lie at the geometrical center of the system; hence, the frame of reference centered on the star is not an inertial reference frame. The star is actually in orbit about the center of mass (i.e. the star is accelerating) and creates an effective forcing potential - the "indirect potential". Our results show that this indirect potential is essential for the growth and maintainence of spiral modes with azimuthal wavenumber $m=1$. In fact, the interaction of this indirect potential with the outer Lindblad resonance in the disk can be the dominant amplification mechanism for these modes (see ARS and STAR).

\subsection{Wave Physics and Spiral Instabilities in Gaseous Disks}

In the simplest description of spiral instabilities, self-excited disturbances (spiral modes) can grow through the feedback and amplification of spiral density waves. 
In this section, we review the theory of spiral density waves. In the following sections, we describe the feedback cycle and the amplification mechanism for spiral modes with $m=1$.

In the asymptotic (WKBJ) limit, the dispersion relation for spiral density waves in a gaseous disk has the form

$$
k^{2} a^{2}-2 \pi G \sigma_{0}|k|+\kappa^{2}=(\omega-m \Omega)^{2},
$$

where $k$ is the radial wavenumber, $\kappa$ is the epicyclic freqeuncy, and where $\omega$ is the complex eigenvalue of the system (see, e.g., Lin \& Lau 1979). Since the gravitational term is proportional to $|k|$, this dispersion relation has four branches,

$$
k= \pm\left(k_{0} \pm k_{1}\right)
$$

where

$$
k_{0} \equiv \frac{\pi G \sigma_{0}}{a^{2}}, \quad k_{1} \equiv \frac{\pi G \sigma_{0}}{a^{2}}\left[1-Q^{2}\left(1-\nu^{2}\right)\right]^{1 / 2}, \quad \text { and } \quad \nu \equiv(\omega-m \Omega) / \kappa .
$$

Here, the quantity $Q$ determines the stability of the system to axisymmetric disturbances $(Q>1 \Rightarrow$ axisymmetric stability) and is defined by (Toomre 1964):

$$
Q \equiv \frac{\kappa a}{\pi G \sigma_{0}} .
$$

The overall sign of $k$ determines whether the waves are leading $(|k|>0)$ or trailing $(|k|<0)$; the inner sign determines whether the waves are short $[k \propto$ $\left.\left(k_{0}+k_{1}\right)\right]$ or long $\left\{k \propto\left(k_{0}-k_{1}\right)\right\}$.

The quantity $\nu$ is a dimensionless frequency of the spiral density waves. The radius in the disk where $\nu=0$ (i.e. where $\Re(\omega)=m \Omega$ ) is known as the corotation resonance (CR); the energy and angular momentum of the perturbation (and the action) are positive outside the corotation radius and negative inside. Notice that for $Q>1$, the wavenumber $k_{1}$ becomes imaginary for radii sufficiently close to the $\mathrm{CR}$, i.e. a classical turning point exists for the density waves. The resulting "forbidden" region surrounding the CR is known as the " $Q$-barrier". Notice also that for long waves, $k \rightarrow 0$ at any radius where $|\nu|=1$. The radius in the disk where $\nu=+1$ is known as the outer Lindblad resonance (OLR) and plays an important role in the physics of $m=1$ modes.

\subsection{Feedback Loop: The Four-Wave Cycle}

We now present the feedback cycle for $m=1$ modes in gaseous disks (from STAR). One unique aspect of this feedback cycle is that all four types of waves are utilized (see Figure 1):

1. Begin (somewhat arbitrarily) with the excitation of a long trailing (LT) spiral density wave at the outer Lindblad resonance (OLR) by the indirect term. The LT wave propagates inward (its group velocity is negative) until it encounters the outer edge of the $Q$-barrier.

2. At the $Q$-barrier, the LT wave refracts into a short trailing (ST) spiral density wave that propagates back outward, through the OLR to the outer disk edge. 


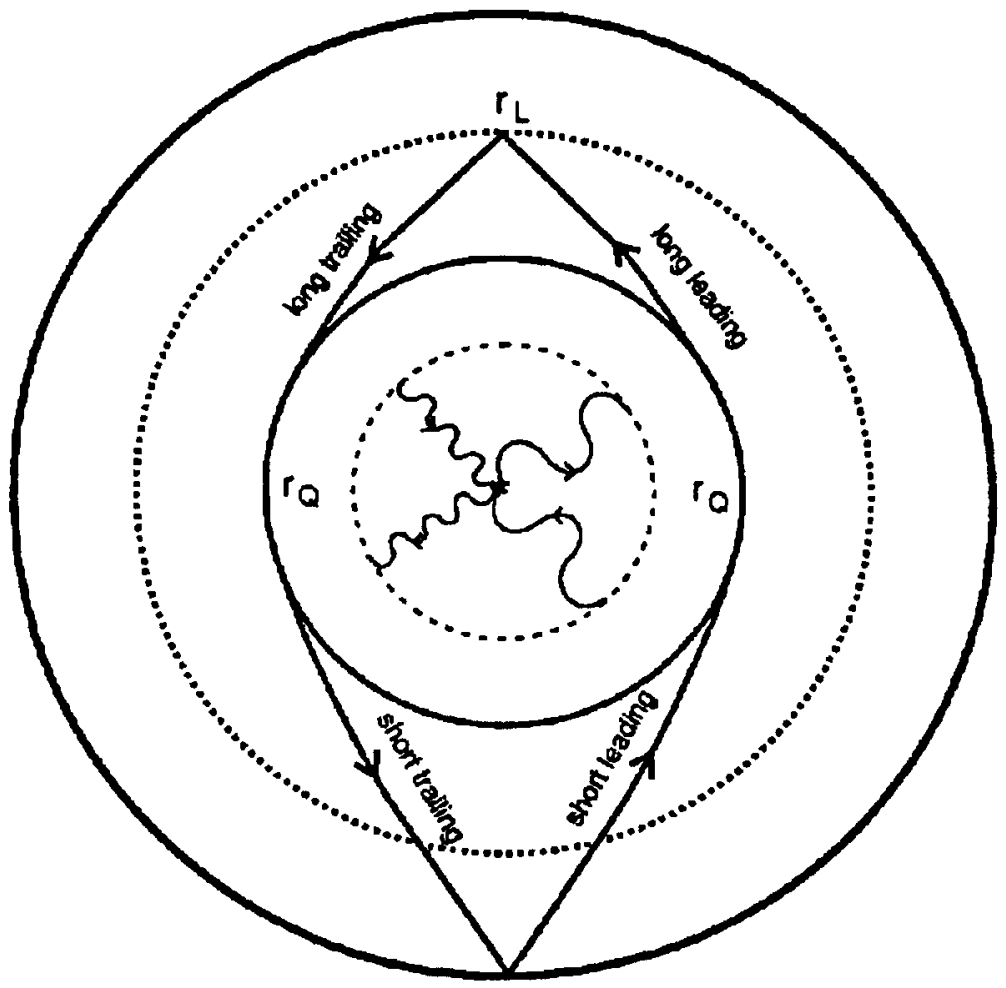

rD

FIGURE 1. Four wave cycle

3. The ST waves that propagate to the outer disk edge reflect there to become short leading (SL) waves. The SL waves then propagate back to the interior, through the OLR, until they encounter the outer edge of the $Q$-barrier, where they refract into long leading (LL) spiral density waves that propagate back toward the OLR.

4. At the OLR, the LL waves reflect to become LT waves. If the reflected LT wave possesses the correct phase relative to the LT wave launched from OLR by the indirect term in step 1 above, then we have constructive reinforcement of the entire wave cycle, and the basis for the establishment of a resonant wave cavity.

Using a WKBJ analysis, we have derived a quantum condition on the basis of the above four-wave cycle. This quantum condition accurately predicts the pattern speeds (i.e. the real part of the eigenfrequencies) for these modes; for strongly growing modes, the analytical and numerical results agree to within $\sim 1$ percent (see STAR). 


\subsection{SLING Amplification}

In this section, we describe the amplification mechanism for eccentric modes. The analysis indicates that the dominant mechanism for amplification arises from the indirect potential, which provides an effective forcing term. The indirect term varies slowly with radius in the disk; since a slowly varying force can only couple to oscillatory disturbances at the disk edges or at the Lindblad resonances, the main coupling occurs at the outer Lindblad resonance for the modes considered here. Thus, this amplification mechanism differs substantially from the previously studied mechanisms, which utilize the process of super-reflection across the corotation resonance (super-reflection can still occur in these disks and is included in the numerical treatment, but it does not dominate the amplification). In our analytic treatment, we determine the growth rates for the modes under the assumption that all of the amplification arises from this coupling of the indirect term to the outer Lindblad resonance in the disk (and that the indirect term arises mostly near the outer disk edge). In other words, we conceptually regard the indirect potential as an external forcing term acting on the disk and calculate the torque exerted on the disk at the OLR. Since the long-range coupling of the star to the outer disk provides the essential forcing, this new instability mechanism is called SLING: Stimulation by the Long-range Interaction of Newtonian Gravity.

The combined numerical and analytical treatments indicate the dependence of the growth rates (i.e. the imaginary part of the eigenfrequencies) on the parameters of the problem. Most importantly, a finite threshold exists for the SLING amplification mechanism. When all other properties of the star/disk system are held fixed, this effect corresponds to a threshold in the ratio of disk mass $M_{D}$ to the total mass $M_{*}+M_{D}$. We find that the growth rates are largest for the case of equal masses $M_{D}=M_{*}$ and decrease rapidly with decreasing relative disk mass. In the optimal case, $M_{D}=M_{*}$, the grow rates can be comparable to the orbital frequency at the outer disk edge, i.e. the modes can grow on nearly a dynamical timescale. On the other hand, the presence of the finite threshold implies a critical value of the relative disk mass, i.e. the maximum value of $M_{D} /\left(M_{*}+M_{D}\right)$ that is stable to $m=1$ disturbances; for the simplest case of a perfectly Keplerian disk and $Q\left(R_{D}\right)=1$, this critical ratio has the value $M_{D} /\left(M_{*}+M_{D}\right)=3 / 4 \pi$.

\section{NONLINEAR SPH SIMULATIONS OF DISK INSTABILITIES}

In order to study the nonlinear behavior of the gravitational instabilities which are suggested by the linear analysis, we have performed smooth particle hydrodynamic ( $\mathrm{SPH}$ ) calculations of the behavior of these instabilities in nearly Keplerian disks (Benz \& Adams 1992). Previous studies have performed $N$ body simulations of similar star/disk systems (e.g., Tomely et al. 1991) and have obtained qualitatively similar results.

\subsection{The Initial System}

In an ideal case, we would like our initial, unperturbed systems to be as close as possible to real star/disk systems found in star forming regions (recall that 
the linear stability analysis of the previous section adheres to this goal as closely as possible). In the present case of nonlinear simulations, however, we cannot reproduce the required dynamic range in radial extent, i.e. $R_{D} / R_{*} \sim 10^{4}$. For the simulations described here, we are limited to the somewhat smaller range of $R_{D} / R_{\text {in }}=60$. However, because the gravitational potential of the central "star" is softened to avoid divergences, the range of radii for which the dynamics is not perturbed by numerical effects reduces to $R_{D} / R_{i n}=30$. The inner radius $R_{\text {in }}$ of the simulation does not correspond to the stellar radius, but rather to an inner cutoff radius at which we can apply a suitable inner boundary condition (see below).

For the remainder of the system parameters, we can adopt values which are in accordance with those expected in real astrophysical systems. In this study, we consider the ratios of the disk mass to the stellar mass to lie in the range $1 / 2 \leq M_{D} / M_{*} \leq 1$. The initial temperature distribution of the gas in the disk is taken to be a power-law in radial distance; we generally adopt the power-law form $T \sim r^{-1 / 2}$ appropriate for the so-called "flat-spectrum" sources. Although little is known about the surface density distributions in real astrophysical disks, we adopt a power-law form $\sigma \sim r^{-3 / 2}$ in accordance with theoretical considerations (e.g., Cassen \& Moosman 1981) and in agreement with the linear calculations (ARS, STAR).

\subsection{The Simulations}

The numerical simulations were performed using the smooth particle hydrodynamics code written by $W$. Benz. All of the simulations run so far have used the two dimensional version of the code. Since space is limited in this present volume, we refer the reader to previously published descriptions of the code characteristics (Benz 1990; see also Benz et al. 1990). For the simulations of star/disk systems considered here, the number of particles $N$ was taken to be in the range $N=6000-20,000$ (these values of $N$, which were determined by the available computing resources, adequately resolved disks with the ratio of outer to inner radii of 30 ). All of the present simulations use an isothermal equation of state, i.e. $p=a^{2} \rho$, where the sound speed $a$ is a constant. From a physical point of view, this assumption implies that the disk is able to radiate all energy dissipated in shocks or adiabatic compression.

For the inner boundary condition, we allowed the central star to "swallow" all particles coming within a fixed distance (here arbitrarily chosen to be $R_{D} / 50$ ). This ansatz avoided extreme overcrowding in the very central regions of the system and hence avoided an excessively small time step. As the particles are eaten, the mass of the central star is increased correspondingly and the star is displaced to conserve momentum. During the course of a typical simulation, a few percent of the disk mass is eaten in this manner.

The linear stability analysis (ARS, STAR) suggests that modal growth is relatively insensitive to the inner boundary condition, but rather sensitive to the outer boundary condition (the effects of the outer boundary are quantitfied in Ostriker et al. 1992). In particular, the outgoing ST waves must be able to reflect off of the outer edge. For the SPH simulations, we adopted a "free" outer boundary condition, i.e. the particles on the outer edge are free to wander according to the gravitational and pressure forces exerted on them by the rest 
of the system.

Finally, we specify the initial perturbations of the disk. Since we are constrained (by computing resources) to studying only the first few orbits of the outer disk edge (the hence the first $\sim 100-200$ orbits of the inner edge), we start the simulations with $1 \%$ amplitude perturbations in density with azimuthal wavenumber $m=1$. The results of these simulations are described in the following section.

\subsection{Nonlinear Results}

We find that gravitational instabilities can grow strongly in these systems, i.e. the growth rates are comparable to the dynamical timescale of the outer disk edge. For disks which are not too far from the condition of stability to axisymmetric modes, spiral instabilities with azimuthal wavenumbers $m=1,2,3,4$ (and higher) are present. As stability is increased (i.e. as the Toomre $Q$ values are raised or the mass ratio $M_{D} / M_{*}$ is decreased), the relative strength of the $m=1$ disturbance increases, although the growth rates of all modes decrease (as expected). Finally, and perhaps most importantly for this present discussion, we find that a spiral arm can often collapse to form a "knot" of bound gas when the equation of state of the disk is taken to be isothermal (see Figure 2). These collapsed "knots" typically have masses of $\sim 0.01 M_{D}$ and travel on elliptical orbits; in the discussion below, we speculate on the possible long term behavior of these objects. At this point, its necessary to add a note of caution. These knots can be formed as a result of our assumption of isothermal evolution of the disk. This assumption limits the local pressure support available. The density enhancements found in our unstable modes are sufficient for pushing small regions over the local Jeans mass and thus triggering the collapse. This collapse would not occur for adiabatic evolution. Hence, our results bear any connection to real physical systems only to the extent that these systems can radiate away a large fraction of their energy.

\section{DISCUSSION}

We have studied gravitational instabilities in gaseous disks using both numerical and analytical methods. For the linear problem, we have determined the (complex) eigenvalues of the system; the numerical and analytical approaches are in good agreement. This analysis indicates that the basic modal mechanism involves the four-wave cycle (see Figure 1), which provides the feedback loop, and the SLING mechanism, which provides the amplification (see STAR). The linear results also indicate that a wide range of YSO disks will be unstable to the growth of eccentric $(m=1)$ distortions. When the disk mass is comparable to the stellar mass $\left(M_{D} \sim M_{*}\right)$, these distortions can grow on nearly a dynamical timescale. In addition, we find that these modes can grow when the disk is safely stable to axisymmetric disturbances (i.e. $Q$ substantially greater than 1 ). However, we find that our results (e.g., the exact spectrum of unstable modes) are particularly sensitive to the treatment of the outer disk edge (Ostriker et al. 1992).

We have studied the nonlinear evolution of gravitational instabilities using smooth particle hydrodynamic simulations. These studies indicate that self- 


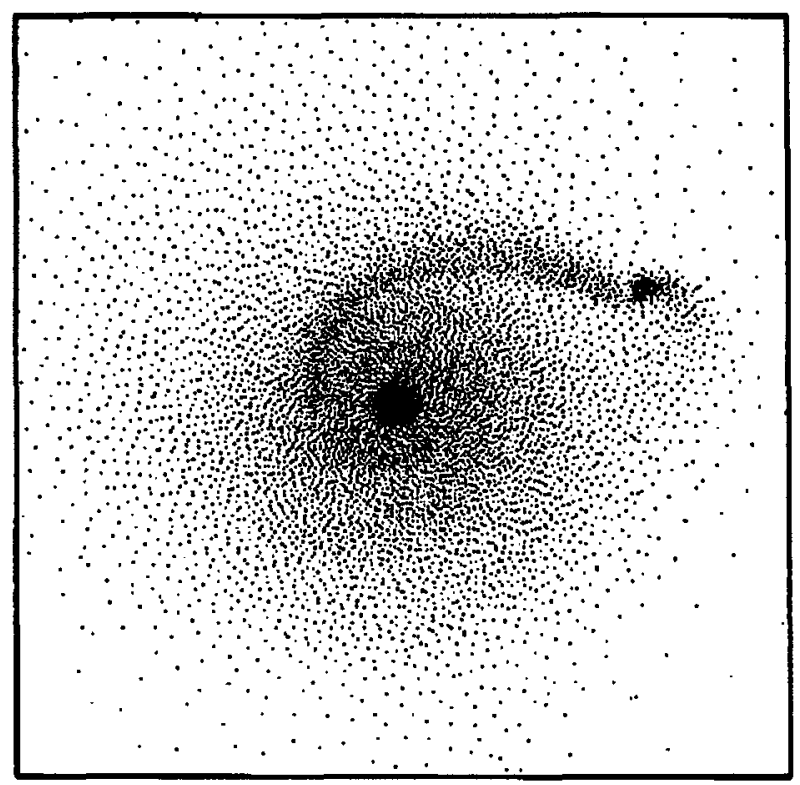

FIGURE 2. Snapshot of an isothermal disk $\left(M_{D}=M_{*}\right.$ and $\left.Q_{\min }=1.75\right)$ after slightly more than one outer edge rotation. Notice the strong $m=1$ mode and the formation of a dense knot.

gravitating instabilities (with $m=1$ and higher) will grow into the nonlinear regime. Under most circumstances, the disk edge does not soften enough that modal growth turns off. In particular, we find that the star continues to spiral away from the center of mass of the system throughout the simulation. In many cases, a spiral arm will collapse into a gravitationally bound "knot" of gas; these knots typically contain $1 \%$ of the mass of the disk and travel on elliptical orbits about the central star.

The instabilities discussed in this work may have important astrophysical applications. In the earliest stage of star formation - the protostellar phase the mass of the disk is likely to be comparable to that of the star (see Shu et al. 1987); $m=1$ modes are thus likely to grow and may lead to mass accretion through the disk and the observed disk luminosities. Perturbations with $m=1$ prove especially interesting because they force the star to move from the center of mass and thereby transfer angular momentum to the stellar orbit. As shown in the nonlinear SPH simulations, this coupling can lead to the production of a "knot" of gravitationally bound gas which orbits the star. The most interesting unresolved question then becomes: Can these "knots" of gas evolve into interesting astrophysical bodies, such as binary companions and/or giant planets, or do they become tidally ripped apart? Although future work is required to settle this issue, we speculate below on the possibilities.

The most likely scenario of binary formation within a disk occurs as follows. The work described above suggests that gravitational instabilities in the disk 
become important when the disk mass becomes comparable to the stellar mass. In the current picture of star formation (as reviewed in Shu et al. 1987), star/disk systems are built up from the inside-out collapse of a molecular cloud core. The low angular momentum material falls first and hence a stellar object forms at the center of the collapse flow. Material with higher angular momentum falls in later and collects in a circumstellar disk. For typical initial conditions in molecular cloud cores, most of the material falls directly onto the disk rather than onto the star. In particular, the disk mass will become comparable to the stellar mass when the total mass of the central star/disk system is still quite small (e.g., $\left.0.05-0.1 \mathcal{M}_{\odot}\right)$ compared to the final mass of the star $\left(\sim 1 \mathcal{M}_{\odot}\right)$. Now suppose that the gravitational instabilities described above occur at this very early stage of evolution. We then have a very small star $\left(\sim 0.05 \mathcal{M}_{\odot}\right)$ with a very very small companion $\left(\sim 0.0005 \mathcal{M}_{\odot}\right)$, with most of the material which will make up the final system yet to come down. Thus, provided that the companion can gain an appreciable share of the newly arriving material, the system can evolve into a typical binary system.

The final issue that we must address is how this scenario for binary formation relates to other possible mechanisms and to the emerging paradigm of star formation. The capture scenario for binary formation is on a different footing and has its own pros and cons (see the review by C. Clarke in this volume). Fragmentation scenarios (see the chapters by A. Boss and I. Bonnell) are qualitatively the same, but differ in the size scale and timing of how a cloud core breaks up into two (or more) pieces. In this scenario of binary formation within a disk, we are considering the limiting case in which cloud material falls all the way into a central star/disk system and then breaks up into multiple parts. One important constraint on these theories is provided by observations of protostellar candidates. The observed spectral energy distributions of these protostars are in good agreement with the current protostellar theory, which includes only a single star (see Adams et al. 1987). In addition, observed maps of the spatial distribution of emission in protostars (Ladd et al. 1991) typically show only a single condensation center and not two (on a size scale of $\sim 2000 \mathrm{AU}$ ). As a result, observations suggest that the breakup of protostars into two components occurs at a relatively small size scale $(<1000 \mathrm{AU})$, whether the breakup occurs via fragmentation or through disk instabilities.

\section{REFERENCES}

Abt, H.A. 1983, ARA\&A, 21, 343
Adams, F.C., Emerson, J.E., \& Fuller, G.A. 1989a, ApJ, 357, 606
Adams, F.C., Lada, C.J., \& Shu, F.H. 1987, ApJ, 312, 788
Adams, F.C., Lada, C.J., \& Shu, F.H. 1988, ApJ, 326, 865
Adams, F.C., Ruden, S.P., \& Shu, F.H. 1989b, ApJ, 347, 959 (ARS)
Appenzeller, I. \& Mundt, R. 1989, ARA $8 A, 1,291$
Benz, W. 1990, in Numerical Modeling of Nonlinear Stellar Pulsations: Problemo and
$\quad$ Prospects (Dordrecht, Kluwer Academic Press)
Benz, W., Bowers, R.L., Cameron, A.G.W., \& Press, W. H. 1990, ApJ, 348, 647
Benz, W. \& Adams, F.C. 1992, in preparation
Bertout, C. 1989, ARA\&A, 27, 351
Cassen, P. \& Moosman, A. 1981, Icarus, 48, 353
Edwards, S., Cabrit, S., Strom, S.E., Heyer, I., Strom, K.M., \& Anderson, E. 1987, 
$A p J, 321,473$

Lada, C. J. \& Shu, F.H. 1990, Science, 1111, 1222

Ladd, E.F., Adams, F.C., Casey, S., Davidson, J.A., Fuller, G.A., Harper, D.A., Myers, P.C., \& Padman, R. 1991, ApJ, 382, 555

Lin, C.C. \& Lau, Y.Y. 1979, Studies in Applied Math., 60, 97

Ostriker, E.C., Shu, F.H., \& Adams, F.C. 1992, $A p J$, in press

Shu, F.H., Adams, F.C., \& Lizano, S. 1987, ARA\&A, 25, 23

Shu, F.H., Tremaine, S., Adams, F.C., Ruden, S.P. 1990, ApJ, 358, 495 (STAR)

Tomley, L., Cassen, P., \& Steiman-Cameron, T. 1991, ApJ, 382, 530

Toomre, A. 1964, ApJ, 139, 1217

\section{DISCUSSION}

LEINERT: Can your proposed mechanism be used to explain the formation of outer planets?

ADAMS: The "knots" of gas that form have roughly the right mass and orbits to become giant planets. However, people who study giant planets claim that such planets cannot be formed through gravitational instability: all of the heavy elements are in the center of the planet and in a configuration which is more suggestive of buildup by aggregation.

BOSS: In order for the clumps to grow to stellar size, you suggested that ongoing accretion of gas might be occurring. Do you think that the infalling gas will have any deleterious effect on the sharp edge of the disk needed for growth of the instability?

ADAMS: No. The infall collapse solution naturally produces an angular momentum barrier and hence a well-defined outer disk edge.

MARSCHALL: You have shown that this mechanism can form pairs of stars, but can it also produce systems of higher multiplicity?

ADAMS: Maybe. Suppose that the instability described here occurs at a fairly small outer disk radius, say $0.1 \mathrm{AU}$. We then form a binary with approximately a $0.1 \mathrm{AU}$ separation. If the infall continues for a long time after that stage, a large circumbinary disk can result. If this disk is sufficiently massive, the instability can grow again (the binary pair now plays the role of the star to produce the indirect potential) and a binary companion can be produced in the outer disk. This thus gives a triple system.

SIMON: Do I understand correctly that this mechanism will tend to make relatively wide binaries (e.g., we believe the massive disks to have sizes of $\sim 100 \mathrm{AU}$ )?

ADAMS: This mechanism can, in principle, form binaries with separations anywhere in the range from the stellar radius to $100 \mathrm{AU}$. The second bodies which form in the disk have orbital radii of roughly half the disk radius at the time the instability sets in. Since the disk can become unstable when its radius is anywhere in the range stated above (this result depends on initial conditions), a full range of binary separations can result. However, it will be hard to make binaries with separations much greater than $\sim 100 \mathrm{AU}$. 